\title{
Anomalous Right Coronary Originating from the Left main Coronary or the mid of Left Anterior Descending Coronary Artery
}

\author{
Xuju Qin ${ }^{1 *}$, Lin Yang2, Weiguo Xiong 3 , Chunpeng Lu3, Xuguang Qin ${ }^{4}$, \\ ${ }^{1}$ Department of Orthopedics Linyi People's Hospital. Linyi 256700, People's Republic of China; \\ ${ }^{2}$ Department of Cardiology, Linshu County's Hospital of Shandong Province. Linshu 276700, People's Republic of China; \\ ${ }^{3}$ Department of Cardiology, the First Affiliated Hospital of Tsinghua University. Beijing 100016, People's Republic of China; blic of China; \\ ${ }^{4}$ Department of Cardiology, Beijing North Asia Orthopedics Hospital. Beijing 102445, People's Republic of China; \\ *Corresponding Author: Xuju Qin, Department of Orthopedics Linyi People's Hospital. Linyi 256700, People's Republic of China. \\ Received Date: November 21, 2020; Accepted Date: January 18, 2021 ; Published Date: January 25,2021 \\ Citation: Xuju Qin Lin Yang, Weiguo Xiong, Chunpeng Lu, Xuguang Qin, (2021) Anomalous Right Coronary Originating from the Left main \\ Coronary or the mid of Left Anterior Descending Coronary Artery. J. Clinical Cardiology and Cardiovascular Interventions, 4(2);
}

Doi:10.31579/2641-0419/113

Copyright: ( 2020 Xuju Qin, This is an open-access article distributed under the terms of the Creative Commons Attribution License, which permits unrestricted use, distribution, and reproduction in any medium, provided the original author and source are credited.

\begin{abstract}
Coronary artery anomalies (CAAs) are very rare a relatively uncommon, diverse group of congenital disorders of coronary arterial anatomy with a clinical presentations. Though most commonly detected incidentally finding during routine catheter, CT angiograms or at autopsy, these anomalies have generated considerable interest as they constitute the second most common cause of sudden cardiac death in young competitive athletes after hypertrophic cardiomyopathy [1]. Their prevalence ranges from $0.2 \%$ to $1.3 \%$ based published series [1-4]. The most common coronary artery anomaly is origination of the left circumflex coronary (LCX) artery from the proximal of right coronary artery (RCA) or right sinus of Valsalva. The second is separate origination of the left anterior descending coronary artery (LAD) and LCX artery from the left sinus of Valsalva. Herein, we present five cases that the anomalous RCA arises from the left main coronary artery or the mid of left left anterior descending coronary artery (LAD). These cases are extremely rare. We bring forth them in an attempt to highlight their significance, and make cardiologist to understand what important the anomalies are, and how to diagnosis and treatment these bifurcation lesions of coronary anomalies.
\end{abstract}

Keywords: coronary anomalies; single coronary anomaly; anomalous right coronary artery

\section{Introduction}

Case 1. An 84 years old man was admitted with chest pain for one month. His coronary risk factors were hypertension and dyslipidemia. The echocardiography showed normal left ventricular function $(\mathrm{EF}=55 \%)$ without regional wall motion abnormality. Coronary angiography was performed through trans-radial approach. The results showed the left main coronary artery was normal. There was $90 \%$ stenosis in the proximal of left anterior descending coronary artery segment, and $70 \%$ stenosis in the proximal of left circumflex artery (LCX). The anomalous right coronary artery (RCA) originates from the proximal of left main coronary artery and $80 \%$ stenosis in the proximal segment (showed in figure 1a-d). A $2.75 \mathrm{X} 22 \mathrm{~mm}$ resolute integrity was implanted in the proximal of LAD segment. He was free of chest pain was treated just oral medical drugs such as aspirin, atorvastatin and benazepril during a 6 months follow-up.

Case 2. A 47-year-young man was admitted to the cardiology department with chest pain for two hours companied with sweating after exertion. His coronary risk factors were hypertension, hyperlipidemia and ex-smoker. Physical examination was normal. Electrocardiography (ECG) showed ST elevation 0.2-0.4mv in leads V1-4. Echocardiography showed normal left ventricular systolic function without regional wall motion abnormality. Coronary angiography was performed through trans-radial access, and only a solitary ostium could be cannulated, which give off left main coronary artery (LMCA), left anterior descending (LAD) artery, left circumflex (LCX) artery, and an anomalous right coronary artery (RCA) originating from the mid of LAD segment (Figure 1a, 1b). The single coronary artery anomaly was classified as type L-II A. The left main coronary artery was patent, but there was $99 \%$ stenosis in the mid segment of LAD, and LCX was normal. There was $50 \%$ stenosis in the ostium of anomalous RCA which originating from the mid of the LAD artery was a dominant (Figure 2a-c).

Based on coronary angiogram findings, the decision was made to proceed with percutaneous coronary interventions (PCI) of the bifurcation lesions to the mid of LAD and the ostium of anomalous RCA using DK-culotte stenting strategy. A 7 Fr sheath was inserted through right radial artery, and the left coronary ostium was engaged with an 7 Fr EBU 3.5 guiding catheter. Two 0.0014 inch balance middle weight (BMW) wires were inserted into the LAD and the anomalous RCA artery. We pre-dilated the LAD and the ostium of anomalous RCA using a $2.5 \times 15-\mathrm{mm}$ Quantum Maverick RX balloon (Boston Scientific Corporation, USA). Thereafter, 
we deployed a 3.0× $23 \mathrm{~mm}$ Firebird (MicroPort Medical Corporation, Shanghai, China) stent to LAD-RCA lesions. Adjunctive post-stenting balloon dilatation was done using a 2.5×15-mm Quantum Maverick RX balloon at mid LAD and a Quantum Maverick RX balloon 3.0×15-mm at the LAD-RCA. We performed DK culotte crushing with a $3.0 \times 18 \mathrm{~mm}$
Firebird (MicroPort Medical Corporation, Shanghai, China) stent at the mid LAD. Additional kissing ballooning was performed using a Quantum Maverick RX balloon $3.0 \times 15-\mathrm{mm}$ at LAD-RCA and a post-stenting balloon Firebird $3.0 \times 18 \mathrm{~mm}$ at the mid LAD. Finally a good angiographic result was seen (Figure. 2d-f).
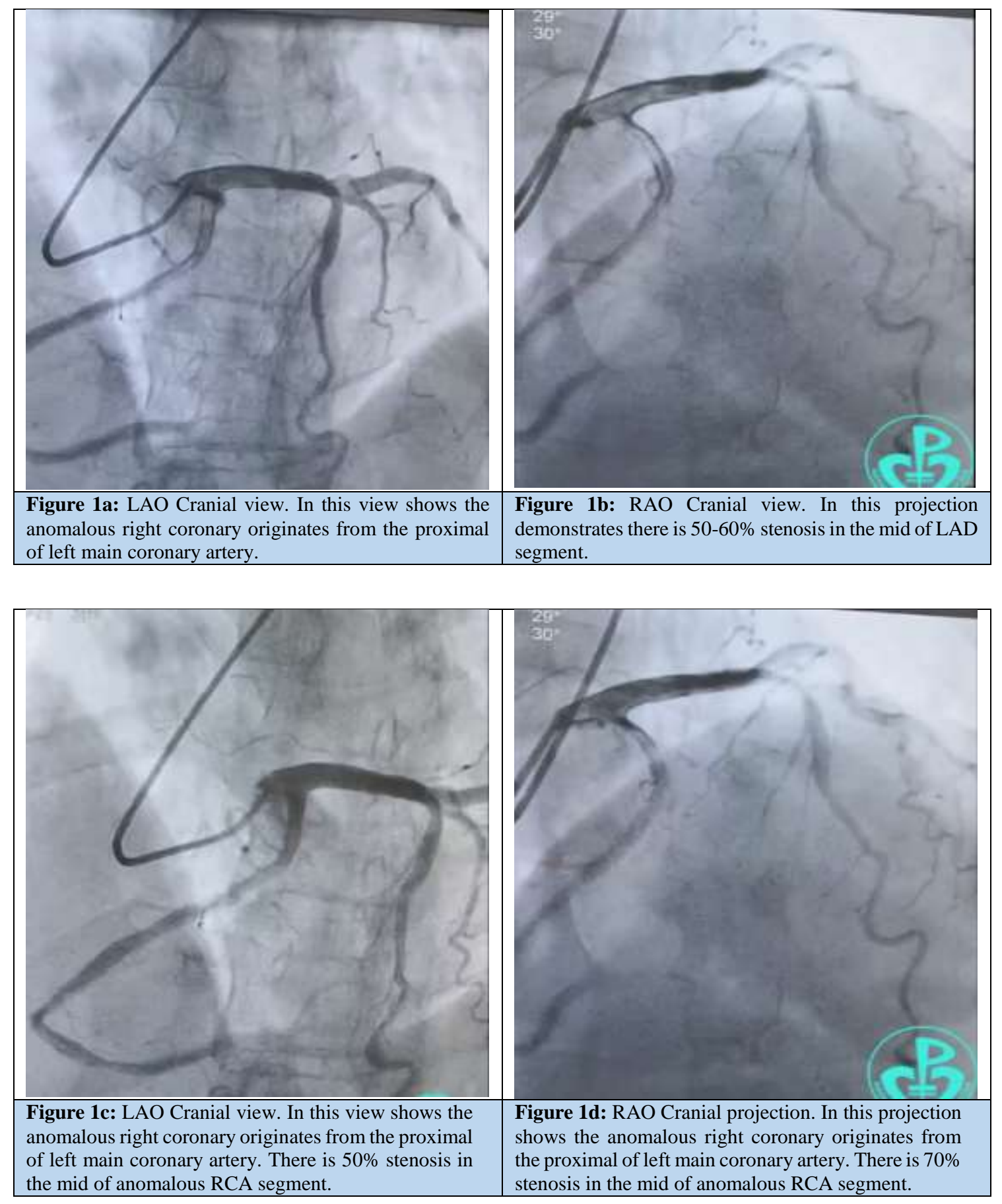


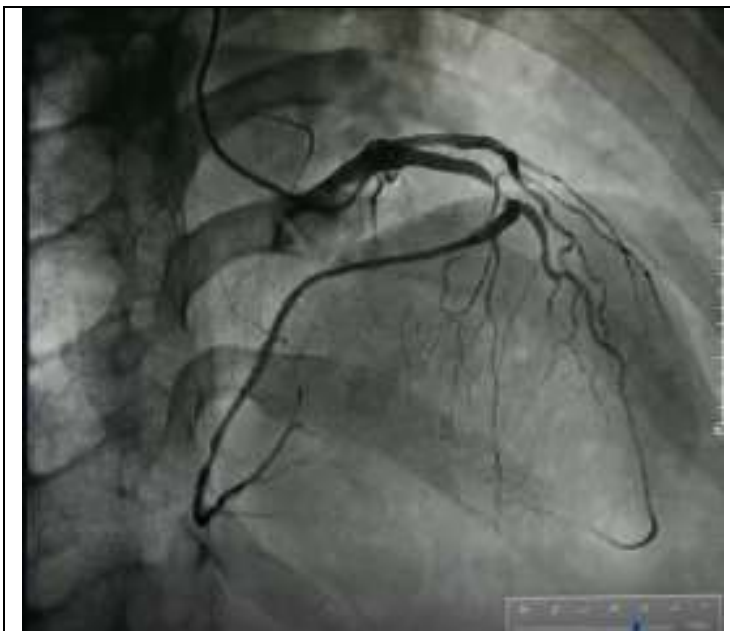

Figure 2a: The AP cranial view. In this view shows there is $99 \%$ stenosis in the mid of LAD segment, and the anomalous RCA originating from the mid of LAD segment.

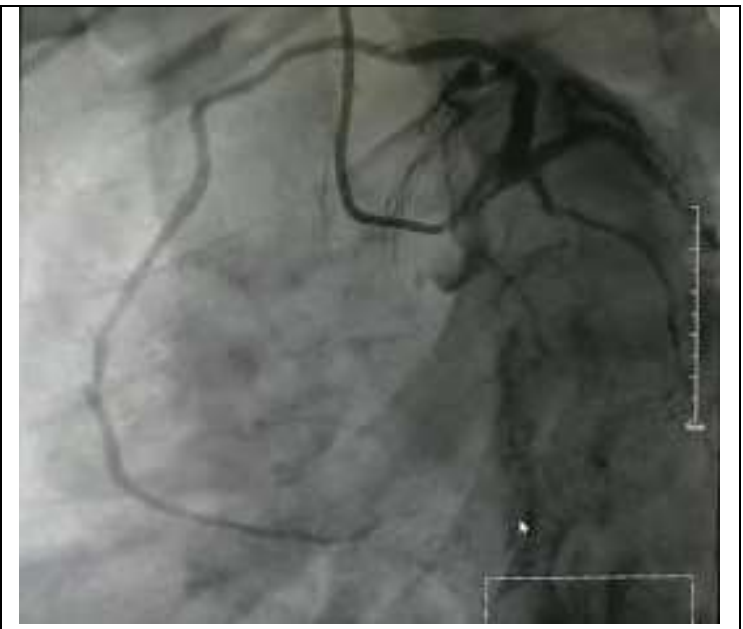

Figure 2b: The LAO caudal view. In this view demonstrates the anomalous RCA originating from the mid of LAD segment.



Figure 2c: The LAO $45^{\circ}$ view. In this view demonstrates no RCA originating from the right sinus Valsalva.

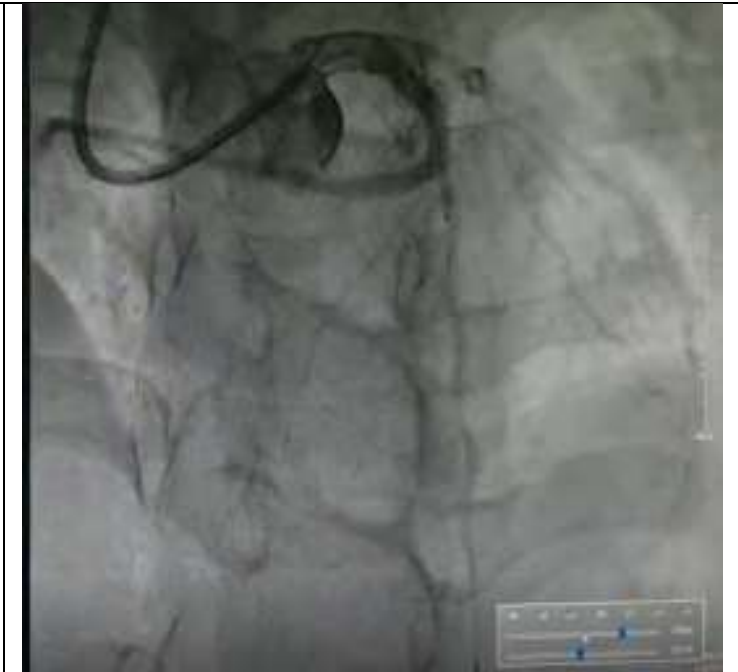

Figure 2d: LAO Cranial view. In this projection shows a $3.0 \times 23 \mathrm{~mm}$ Firebird stent was deployed in LAD-an anomalous RCA segment. 


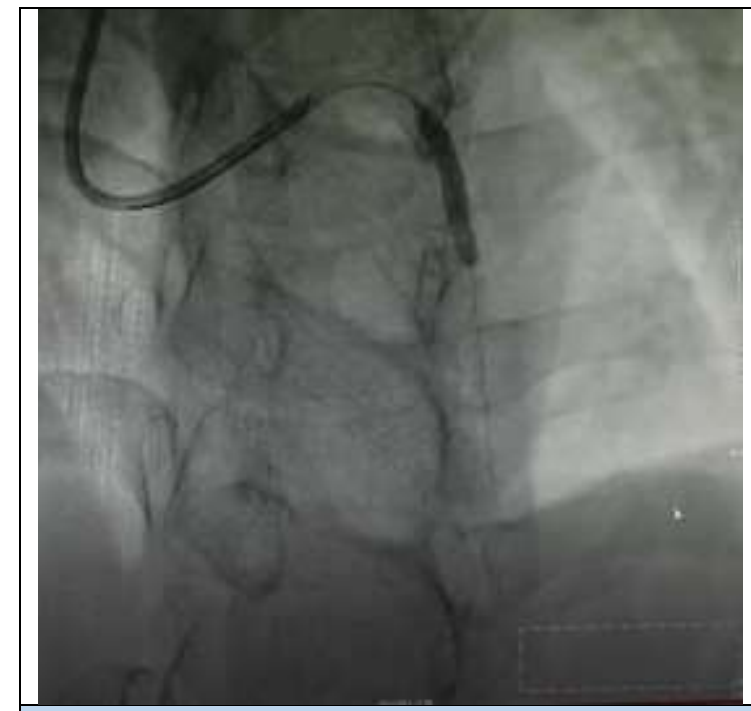

Figure 2e: LAO Cranial view. In this projection shows a $3.0 \times 18 \mathrm{~mm}$ Firebird stent was deployed in the mid of LAD segment.

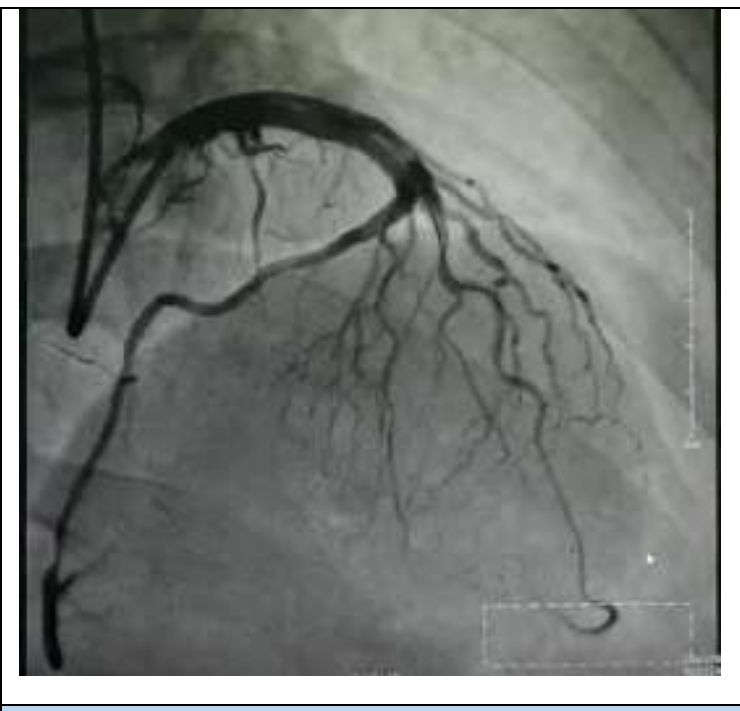

Figure 2f: LAO Cranial view. In this view demonstrates finally a good angiographic result was observed after PCI procedures.
Case 3. A 74-year-old woman presented with a history of progressive angina pectoris for 3 months, she suffered from diabetes mellitus and hypertension for ten years. Coronary angiogram was performed through trans-radial approach. The coronary angiography results showed nonobstructive ostial LMCA and diffuse LAD disease extending from the proximal to the mid segment. The left circumflex artery (LCX) was long lesions in the proximal segment, and the anomalous right coronary artery (RCA) originating from the mid of LAD was patent (showed in figure 3a, 3b).

Based on coronary angiography findings, the decision was made to proceed with percutaneous coronary interventions (PCI) to the proximal and mid LAD and LCX. A 6Fr EBU 3.5 guiding catheter was used to engage the left coronary system. A $180-\mathrm{cm}$ balance middle weight (BMW) wire was used to cannulate the LAD, and the other BMW was used to cannulate the anomalous RCA to protect it. The proximal and mid LAD lesion was predilated with a 2.0-× 20-mm Maverick 2 balloon
(Boston Scientific Corporation, Natic, MA, USA) at 12 atm. A 2.5-× 30 mm Endeavor (Medtronic Corporation, Minneapolis, MN, USA) stent was implanted at the mid LAD segment, and a $3.0-\times 23 \mathrm{~mm}$ Firebird (MicroPort Medical Corporation, Shanghai, China) stent, it crossed over the anomalous RCA, was deployed at the proximal LAD at $14 \mathrm{~atm}$, jailing the wire in the RCA. The mid LAD stent appeared to be underexpanded, so it was postdilated with a $3.0-\times 12-\mathrm{mm}$ Quantum Maverick RX balloon (Boston Scientific Corporation, USA) up to $14 \mathrm{~atm}$. Finally a good angiographic result was observed, and the ostium of anomalous RCA originating from the mid of LAD is not affected (see figure $3 \mathrm{~d}$ ). In order to confirm the origination and course of the anomalous RCA, a 64-slice computed tomography (MSCT) of the heart was performed on a 64-slice machine (Philips 64 Slice, Philips, USA) after 6 months of coronary angiography operation. The results showed the anomalous right coronary artery originating from the mid of the LAD, inferior to the left atrium (Figure 3c). The anomalous RCA was classified as L-II A subtype.

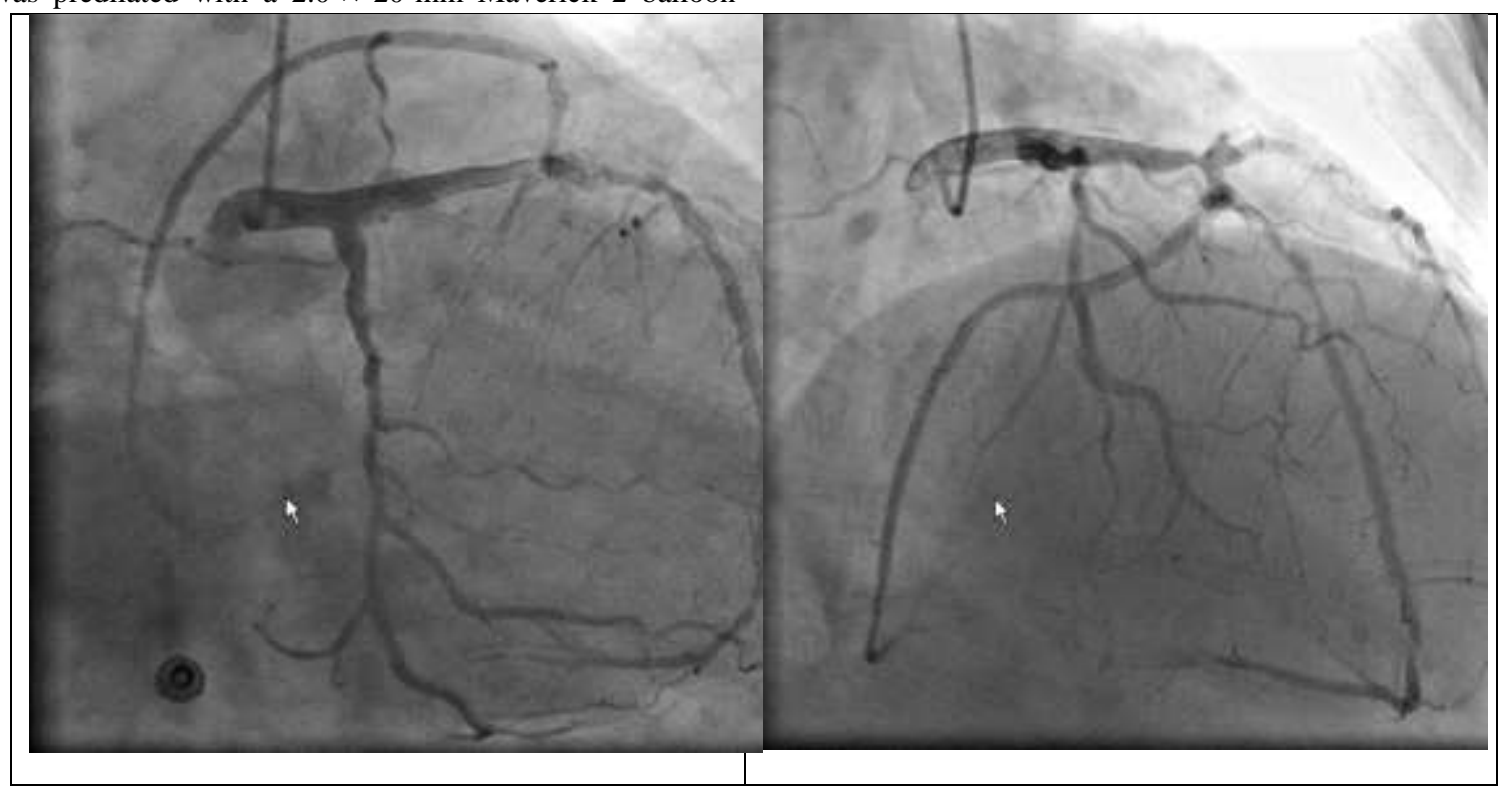


Figure 3a: The RAO caudal view. In this view demonstrates RCA originating from the mid of LAD segment.
Figure 3b: The RAO cranial view shows long lesions in the proximal and mid of LAD segment, RCA arising from the mid of LAD and the lesions of LAD segment.

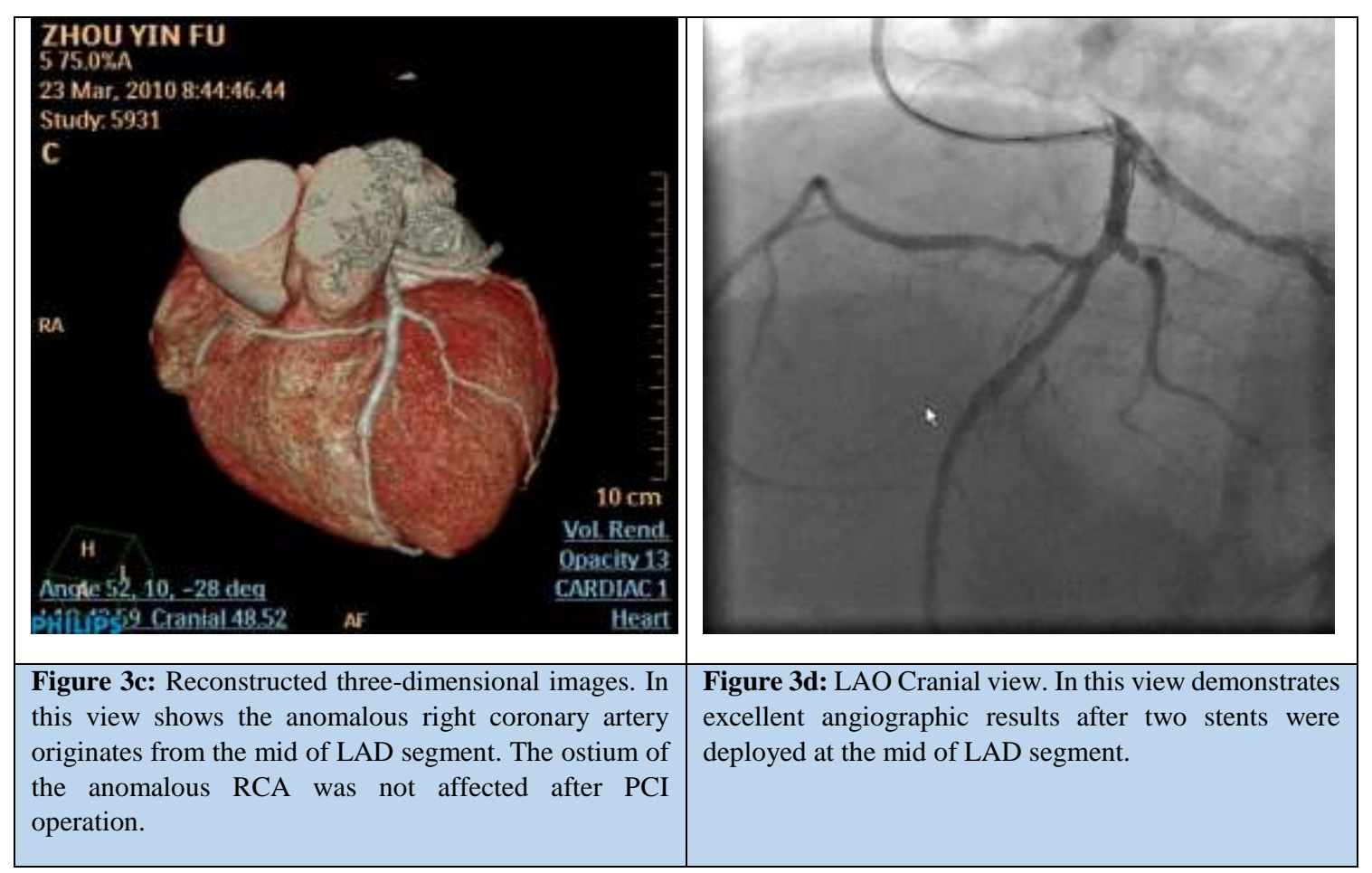

Case 4. A 54-year-old man was admitted with effort chest pain for three months. His coronary risk factors were hypertension and history of exsmoking. The echocardiography showed normal left ventricular function $(\mathrm{EF}=54 \%)$ without regional wall motion abnormality. The physical exam was normal. The ECG showed T-wave inversion in leads V1-4 and chest $\mathrm{X}$-ray was normal. Coronary angiography was performed through transradial access. Left coronary angiography showed the left main coronary was normal. There was $80 \%$ stenosis at the mid LAD segment, while LCX $70 \%$ stenosis in the proximal segment. The anomalous right coronary artery originating from the mid of LAD segment (Figure. 4a-c)

According to coronary angiography findings, the decision was made to proceed with percutaneous coronary interventions (PCI) of the bifurcation lesions to the mid of LAD. A 6Fr Jukin's 3.5 guiding catheter was used to engage the left coronary system. A $180-\mathrm{cm}$ balance middle weight (BMW) wire was used to cannulate the LAD, and the other runthrough (NS) wire was used to cannulate the anomalous RCA to protect it. The mid of LAD lesions was predilated with a 2.5-× 15-mm Maverick 2 balloon (Boston Scientific Corporation, Natic, MA, USA) at 12 atm. A 3.0-× $30 \mathrm{~mm}$ Resolute (Medtronic Corporation, Minneapolis, MN, USA) stent was implanted at the mid LAD segment, it crossed over the anomalous RCA, was implanted at the mid LAD at $14 \mathrm{~atm}$. Finally a good angiographic result was observed (Figure. 4d).

Case 5. A 44-year-old young man was admitted to our hospital with effort chest pain for four months. His coronary risk factors were hypertension and diabetes mellitus. The echocardiography showed normal left ventricular function $(\mathrm{EF}=58 \%)$ without regional wall motion abnormality. The physical exam was normal. The ECG showed T-wave inversion in leads V1-3 and chest x-ray was normal. Coronary angiography was performed through trans-radial access. Left coronary angiography showed the left main coronary was normal, the LAD and LCX were normal. The anomalous right coronary artery originating from the mid of LAD segment, there was $60 \%$ stenosis in the ostia segment (Figure. 4ad). As the lesions of anomalous right coronary artery is borderline, no percutaneous coronary intervention (PCI) and coronary artery bypass graft surgery $(\mathrm{CABG})$ was performed, the patient was just treated oral medical drugs such as aspirin, atorvastatin and acarbosemedically. $\mathrm{He}$ was free of chest pain during a 9 months follow-up. 


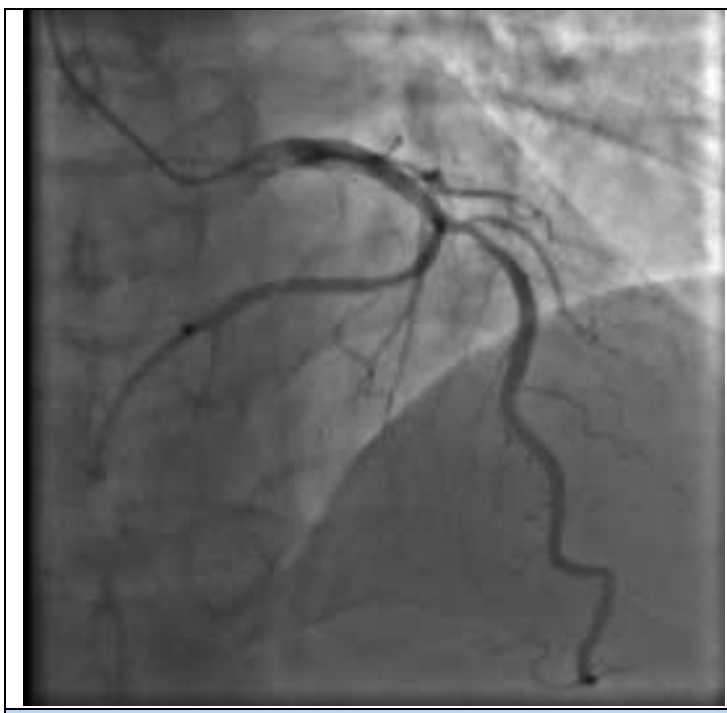

Figure 4a: The RAO cranial view. In this view shows RCA originating from the mid of LAD segment. There was $80 \%$ stenosis in the mid of LAD segment.

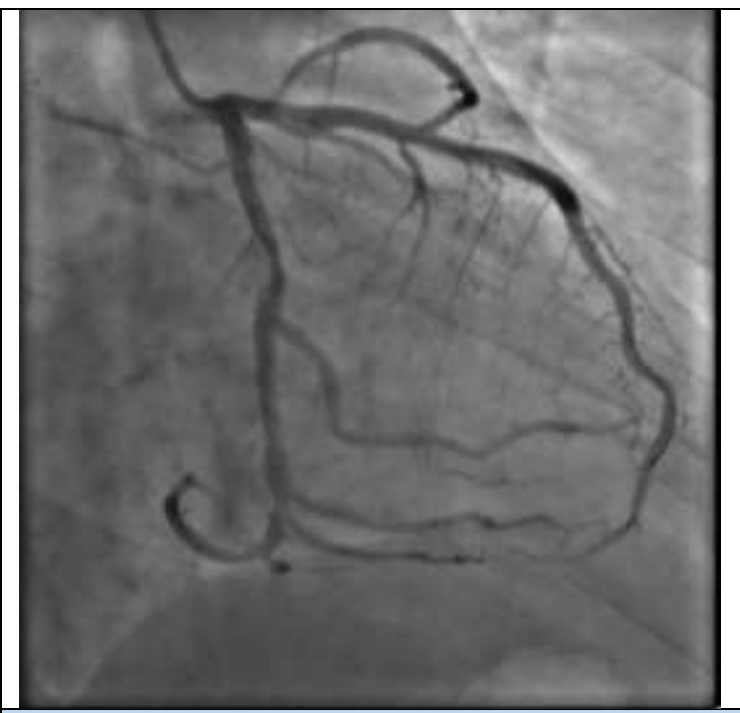

Figure 4b: The RAO caudal view. In this view shows there was $70 \%$ stenosis in the proximal of LCX segment.

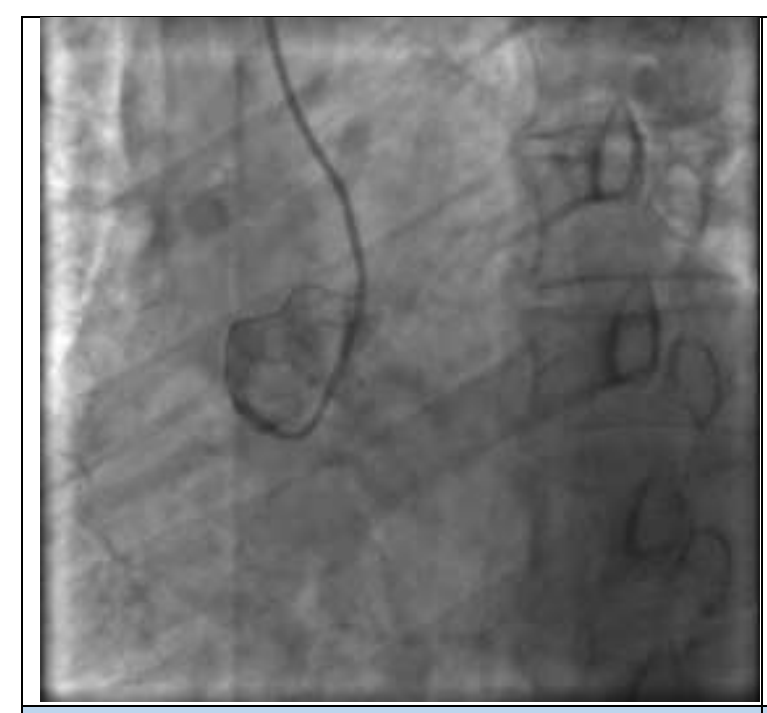

Figure 4c: The LAO view. In this view shows RCA originating from the right sinus of Valsalva.

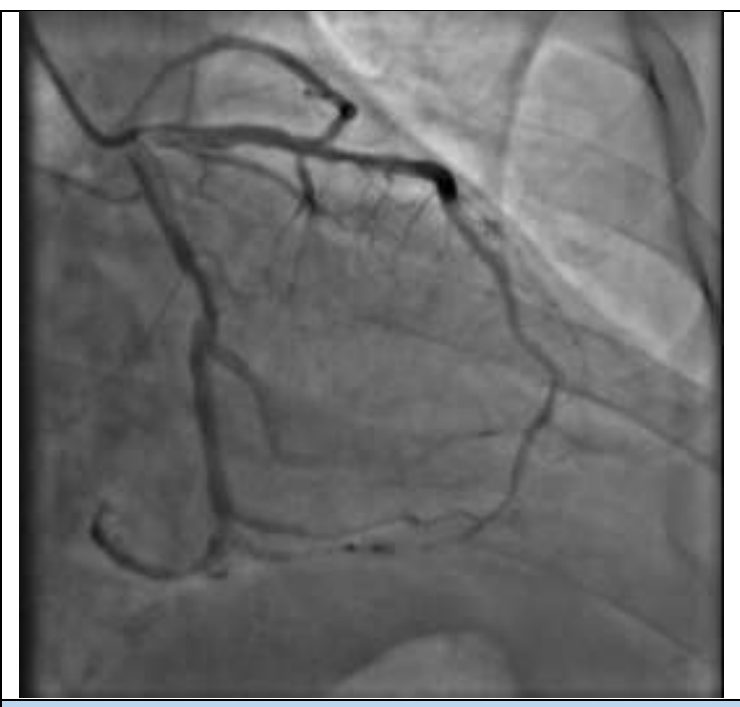

Figure 4d: RAO Caud view. In this view demonstrates the anomalous originates from the mid of LAD segment.

\section{Discussion}

Coronary artery anomalies (CAAs) are very rare a relatively uncommon, diverse group of congenital disorders of coronary arterial anatomy with a clinical presentations. Though most commonly detected incidentally finding during routine catheter, CT angiograms or at autopsy, these anomalies have generated considerable interest as they constitute the second most common cause of sudden cardiac death in young competitive athletes after hypertrophic cardiomyopathy [1]. Their prevalence ranges from $0.2 \%$ to $1.3 \%$ based published series [2-4]. The most common coronary artery anomaly is origination of the left circumflex coronary (LCX) artery from the proximal of right coronary artery (RCA) or right sinus of Valsalva. The second is separate origination of the left anterior descending coronary artery (LAD) and LCX artery from the left sinus of Valsalva. The third is the anomalous right coronary artery (RCA) originating from the aortic trunk of ascending artery or left sinus of Valsalva. When the anomalous right coronary originates from the left main coronary artery or the mid of left anterior descending coronary artery, it is called single coronary artery (SCA) [3], it's prevalence is less than $0.024 \%$ [4]. The anomalous coronary artery is first designated with "R" or "L" depending upon whether the ostium is located in the right or left sinus of Valsalva. It is then designated as group I, II, III. Group I has anatomical course of either a right or left coronary artery. Group II anomalies arise from the proximal part of the normal right or left coronary artery, and cross the base of the heart before assuming the normal position of the inherent coronary artery. Group III describes the anomaly where the LAD and LCX arise separately from the proximal part of the normal right coronary artery $[4,5]$. Herein, we presented five extremely rare cases of anomalous RCA detected incidentally during routine coronary 
angiography. The first case is the anomalous RCA arising from the left main coronary artery (LMCA). When the anomalous RCA originates from the LMCA, it may course between the aorta and pulmonary artery. Although the patient absence of ischemia symptom, he need to be underwent CT angiography to verify the course of anomalous RCA. The other four cases are the anomalous RCA rises from the mid of LAD segment. The second case is bifurcation lesions, the lesions were treated using DK culotte stent technique, finally excellent angiographic results were observed (Figure. 2f). The third and fourth cases are treated using simple provisional stent technique: single stent placement covering the the side branch. Final good angiographic results were obtained (Figure. 3d, 4f).

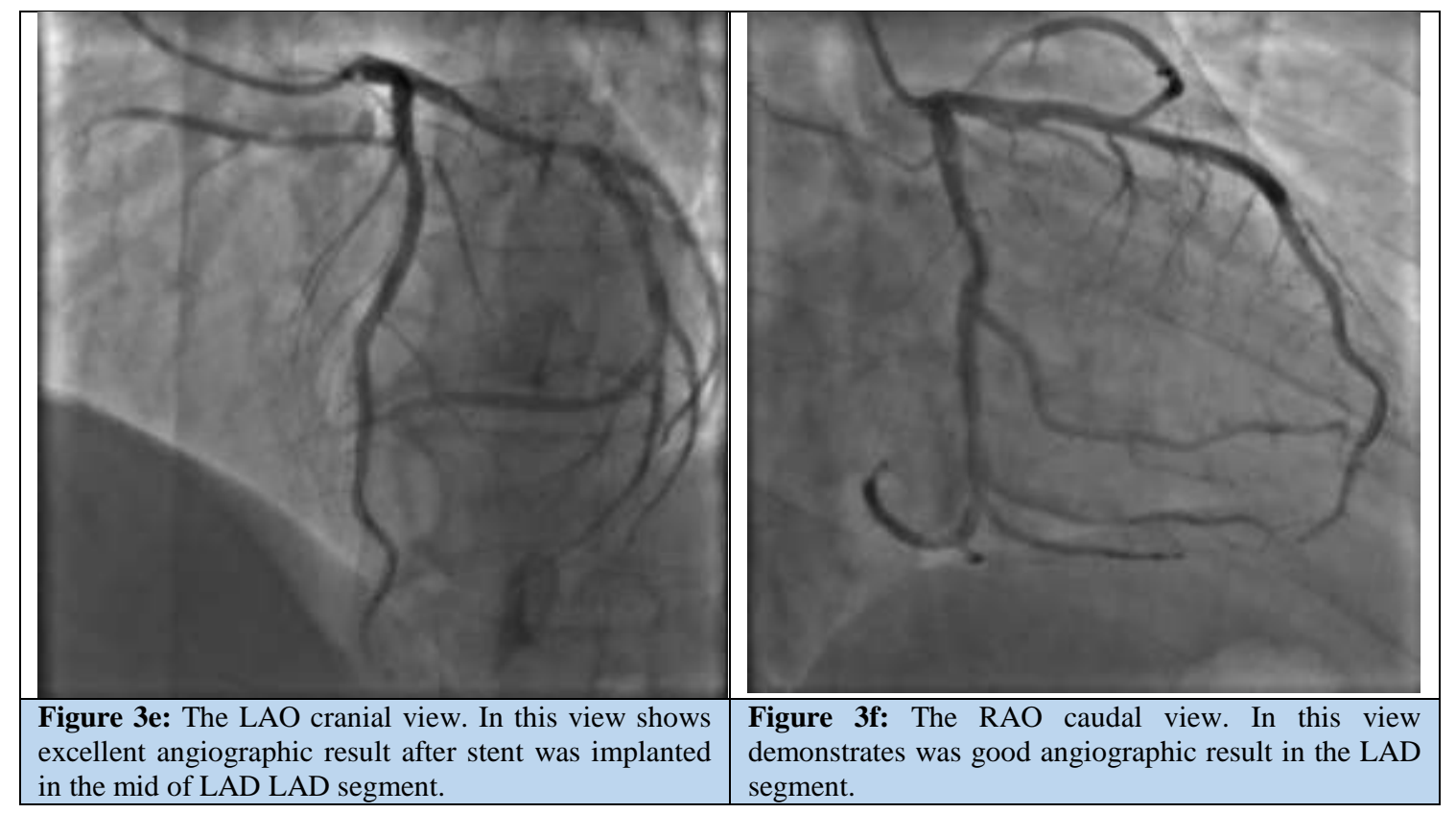

The last four cases are belongs to L-IIA subtypes according to Lipton's classification [5].

The case three, in order to confirm the origination and course of the anomalous RCA, a 64-slice computed tomography (MSCT) of the heart was performed on a 64-slice machine (Philips 64 Slice, Philips, USA) after 6 months of coronary angiography operation. The results showed the anomalous right coronary artery originating from the mid of the LAD, inferior to the left atrium (Figure 3c). The anomalous RCA was classified as L-II A subtype.

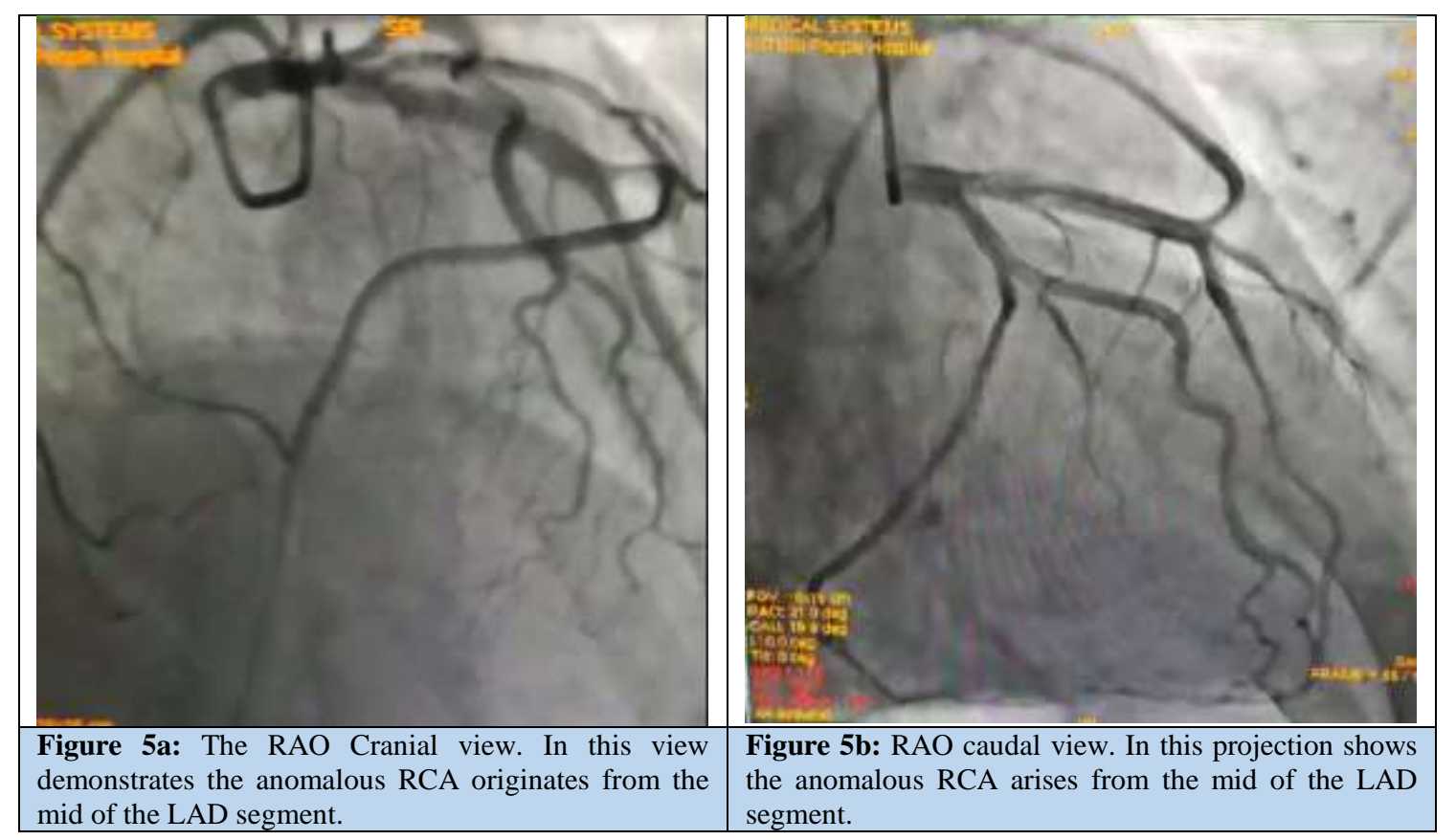




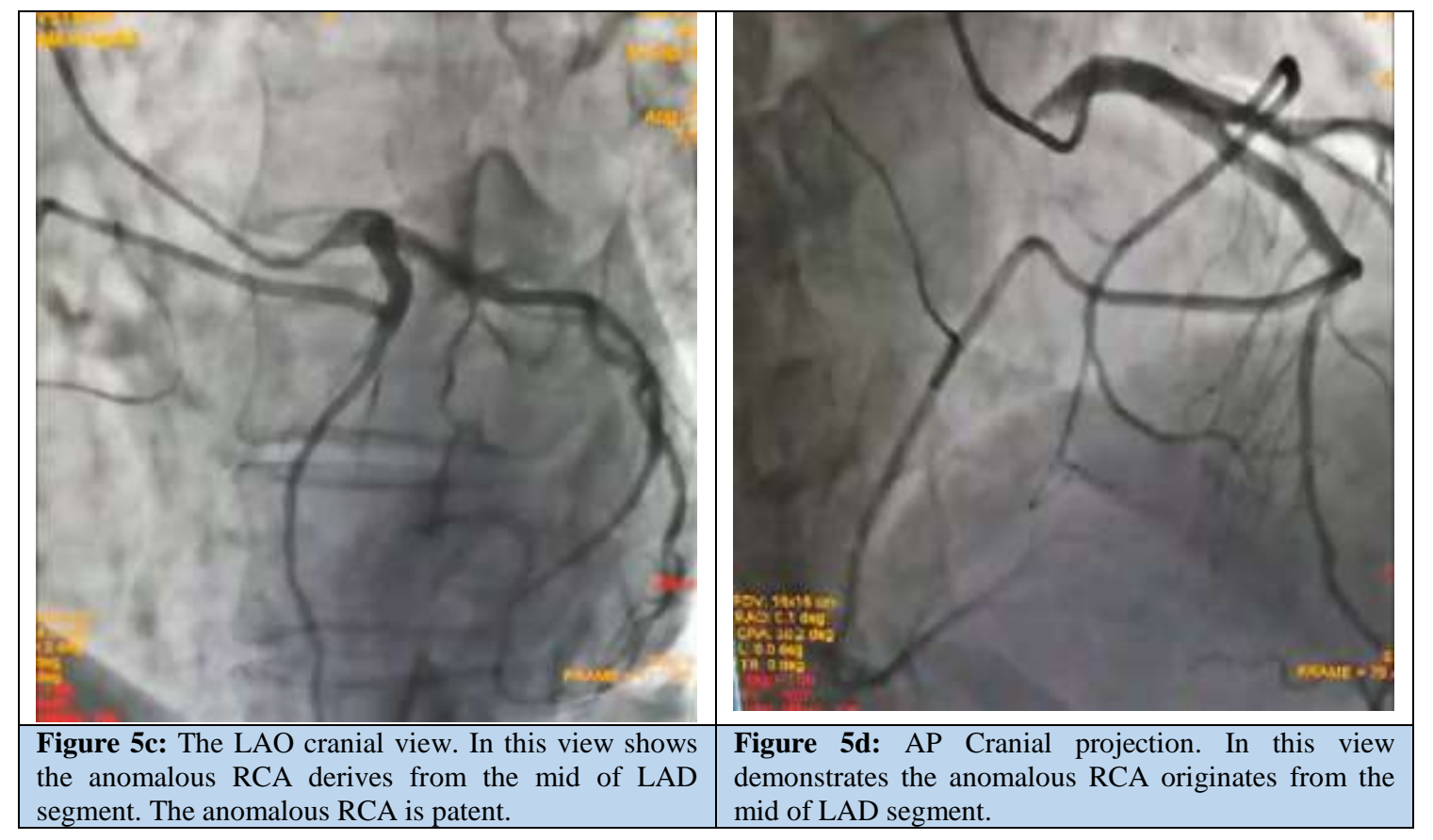

Despite advances in the field interventional cardiology, the optimal stenting technique for bifurcation disease remains the subject of debate. Bifurcation disease accounts for $15-20 \%$ of coronary interventions. According to the 2011 American College of Cardiology (ACC) guidelines, provisional side branch stenting should be the initial strategy (class I recommendation) when the side branch is not large and has only mild to moderate ostial disease, while elective side branch stenting is reasonable (class IIA) if the side branch is large with a high risk of occlusion and the likelihood of side branch reaccess is low [6]. The cases we present is that the anomalous right coronary originates from the LMCA or the mid of left anterior descending coronary artery, and the bifurcation lesions were treated using simple provisional stent or twostent strategy (DK-culotte), final excellent angiography results were

\section{References:}

1. Basso C, Maron BJ, Thiene G. Clinical profile of congenital coronary artery anomalies with origin from wrong aortic sinus leading to sudden cardiac death in young competitive athletes. J Am Coll Cardiol. 2000; 35(6):1493-1501.

2. Yildiz A, Okcum B, Peker T, Arslan C, Olcay A, Bulent Vatan M. Prevalence of coronary artery Anomalies in 12,457 adult patients who underwent coronary angiography. Clinical cardiology 2010; 33:E60-64.

3. Yamanaka O, Hobbs RE. Coronary artery anomalies in 126,595 patients undergoing coronary Arteriography. Cathet Cardiovasc Diag 1990; 21: 28-40.

4. Lipton MJ, Barry WH, Obrez I, Silverman JF, Wexler L. Isolated single coronary artery:

5. diagnosis, angiographic classification, and clinical significance. Radiology 1979; 130: 39-47.

6. Angelini P, Velasco JA, Flamm S. Coronary anomalies. Incidence, pathophysiology, and clinical relevance. Circulation 2002; 105: observed. These cases are extremely rare, so we bring forth them to attempt their significant in our clinical practice [7].

Coronary anomalies are usually detected during coronary angiography. However, X-ray angiography is limited by its inability to provide information regarding the spatial orientation of the anomalous artery with regarding to the surrounding cardiovascular structures [7,8,9]. MSCT coronary angiography has proven to be effective not only in the identification and characterization of coronary artery disease, but also in the evaluation of the anatomical coronary details, and clearly demonstrate the surrounding cardiovascular structures of the anomalous coronary artery.

$$
\text { 2449-2454. }
$$

7. Levine GN et al. 2011 ACCF/AHA/SCAI Guideline for Percutaneous Coronary Intervention: a report of the American College of Cardiology Foundation/American Heart Association Task Force on Practice Guidelines and the Society for Cardiovascular Angiography and Interventions. Circulation. 2011;124D23]:e574-651.

8. He D, Xiong W, Qin X. coronary artery anomalies: the left main coronary artery or left anterior descending coronary artery originating from the proximal of right coronary artery.

9. Chinese Medical Journal.2014;127(12): 2392-2394.

10. Shi, H., Aschoff, A.J., Brambs, H.J. and Hoffmann, M.K. Multislice CT Imaging of Anomalous Coronary Arteries. European Radiology, 2004; 14, 2172-2181.

11. Schmitt, R., Froehner, S., Brunn, J., Wagner, M., Brunner, H., Cherevatyy, O.. Congenital Anomalies of the Coronary Arteries: Imaging with Contrast-Enhanced, Multidetector Computed Tomography. European Radiology, 2005; 15, 1110-1121. 
This work is licensed under Creative Commons Attribution 4.0 License

\section{To Submit Your Article Click Here: Submit Manuscript}

DOI: $10.31579 / 2641-0419 / 113$
Ready to submit your research? Choose Auctores and benefit from:

* fast, convenient online submission

* rigorous peer review by experienced research in your field

* rapid publication on acceptance

* authors retain copyrights

* unique DOI for all articles

* immediate, unrestricted online access

At Auctores, research is always in progress.

Learn more www.auctoresonline.org/journals/clinical-cardiology-andcardiovascular-interventions 